\title{
Information and Symmetry: Adumbrating the Abstract Core of Complex Systems
}

\author{
Lin Bi ${ }^{1}$, Abir U. Igamberdiev ${ }^{2}$ and Pedro C. Marijuán ${ }^{3, *}$ \\ 1 International Research Center for Philosophy of Information, Xi'an Jiaotong University, Xi'an 710049, China; \\ bilin1001@qq.com \\ 2 Department of Biology, Memorial University, St. John's, Newfoundland, A1B 3X9, Canada; \\ igamberdiev@mun.ca \\ 3 Bioinformation Group, Aragon Health Science Institutes (IACS/ISS), 50009 Zaragoza, Spain \\ * Correspondence: pcmarijuan.iacs@aragon.es
}

Academic Editor: Willy Susilo

Received: 6 March 2017; Accepted: 6 March 2017; Published: 14 March 2017

\begin{abstract}
Information and symmetry are essential theoretical concepts that underlie the scientific explanation of a variety of complex systems. In spite of clear-cut developments around both concepts, their intersection is really problematic, either in fields related to mathematics, physics, and chemistry, or even more in those pertaining to biology, neurosciences, and social sciences. The present Special Issue explores recent developments, both theoretical and applied, in most of these disciplines.
\end{abstract}

Keywords: information; symmetry; symmetry-breaking; complex systems; retrocausality; chemical information theory; geno-logical coding; metastable brain; multisensory integration; Planckian information

\section{Introduction}

The present Special Issue on information and symmetry follows from the topical session on Information and Symmetry organized at the Symmetry Festival 2016, celebrated in Vienna, 18-22 July 2016. With this publication we try to gather the most interesting themes presented in that session and to reflect the lively exchanges that took place there. It is also an occasion to deepen our understanding of the relationships between two of the most vexing concepts in theoretical science. In some sense, information and symmetry are "complementary" and the former can be interpreted as the irregular residue that cannot be subsumed under the constructive operations of the latter. However, at the same time, they become "supplementary" and identify two alternative routes of analysis that can be followed to ascertain the underlying structures of complex processes. Information provides diversity metrics and communicative openness, while symmetry provides regular constructive compression and ordering of processes. We are free to alternate them so to ascertain the core complexity of our systems - in general, symmetry breaking provides the most direct link to interconnect them so, for instance, that the passage from a lower level of organization to a higher one may be explained. However, too many factors of a spatial, temporal, structural, compositional, and functional nature may be involved in the dynamics and evolution of most complex systems, so that the way we established the explanatory background will strongly condition the obtainable results. In point of fact, interconnecting heterogeneous explanatory frameworks is the essential problem of today's interdisciplinary and multidisciplinary research programs. In addition, it is precisely in the harmonization of those heterogeneous outcomes where symmetry and information approaches show their highest utility.

Approaches to symmetry range from the most formal fields in mathematics (spatial relationships, geometric transformations, functional transformations, logics-and above all, group theory) to applications in theoretical physics, where invariance (Noether's theorem), symmetry-breaking, internal 
symmetries, and supersymmetries are fundamental [1-3]. Although the relationships with information themes already appear in these formal realms, it is when we go to chemistry, biomolecular fields, physiology, evolution, and social communicative interactions that information and symmetry most clearly have to form an essential tandem ([4,5]; see most of the contributions in this Special Issue too). As can be easily appreciated in these works, information in and of itself cannot be reduced to the Shannonian approach, even more narrowly called a "mathematical theory on communication". Enlarging it is a necessary task, evidently to be conflated with the symmetry paradigm. However, the attempt to transcend towards a more inclusive "foundations of information science" (FIS) approach has shown more difficulties than initially expected [4,6-8]. No doubt that a deep interrelationship of information science and the nascent information philosophy field should exist [9].

\section{Contributions of the Present Issue}

Broadly speaking, the contributions presented herein may be grouped into three main multidisciplinary categories: physico-chemical, biomolecular, and neuroscientific.

\subsection{Information and Symmetry in Physico-Chemical Domains}

We begin with a conjecture on the nature of information developed by Stanley Salthe, where information appears as a result of interactions between observers. Starting with a simple physical example built with forces standing in for observers, the author considers the relation between investigative work and energy constraints, which in turn leads toward a surprising suggestion concerning the most general motivation for work.

Arturo Tozzi and James Peters' work is focused on symmetries, information, and the Monster group before and after the Big Bang. The Monster group, the biggest of the sporadic groups, is equipped with the highest known number of dimensions and symmetries. Taking into account variants of the Borsuk-Ulam theorem and a novel topological approach cast in a physical fashion, the universe may be conceived as a lower-dimensional manifold encompassed in that Monster group. Our universe might arise from a spontaneous dimension decrease and symmetry-breaking which occur inside the very structure of the Monster module.

Koichiro Matsuno develops the theme of retrocausality in quantum phenomena and chemical evolution. An example of empirical evidence demonstrating the significance of retrocausality going along with time-reversal symmetry is seen in the operation of a reaction cycle to be expected in chemical evolution. Quantum mechanics in and of itself has potential in raising a reaction cycle in the prebiotic phase of chemical evolution, even without any help of artefactual scaffoldings of an external origin.

Jerry Chandler analyzes the foundations of chemical information theory. Organic mathematics is an applied mathematics of philosophical atomism. The order of the chemical elements in the table of elements is the source of order for the logical operations of addition and subtraction of atomic numbers. The inverse square laws of physics are the source of organization of subatomic structures of chemical atoms (atomic and molecular structures). These facts are foundational to the logic of the chemical sciences and are therefore the scientific basis for chemical information theory.

\subsection{Information and Symmetry in Biomolecular Domains}

Starting with biological contributions, Sergey Petoukhov and Elena Petukhova examine symmetries in genetic systems and the concept of geno-logical coding. Their article describes basic pieces of evidence in favor of the existence of the geno-logical code, which exists in parallel with the known genetic code of amino acid sequences but which serves for transferring inherited processes along chains of generations. These pieces of evidence have been obtained through the analysis of symmetries in structures of molecular-genetic systems. The obtained results provide a new approach for mathematical modeling of genetic structures, which uses known mathematical formalisms from 
technological fields of noise-immunity coding of information, binary analysis, logical holography, and digital devices of artificial intellect.

Ivan Stepanyan and Sergey Petoukhov approach the matrix method of representation for the analysis and classification of long genetic sequences. This method uses a set of symmetries of biochemical attributes of nucleotides. It also uses the possibility of presentation of every whole set of $\mathrm{N}$-mers as one of the members of a Kronecker family of genetic matrices. A long nucleotide sequence can be visually represented as an individual fractal-like mosaic or another regular binary-type mosaic. Examples of binary mosaics of long nucleotide sequences are shown, including cases of human chromosomes and penicillins.

Tidjani Négadi analyzes the genetic codes and establishes an inverse symmetry-information relationship. First, the author describes the distributions of amino acids and codons, analyzing the degeneracies in the various known genetic codes. Then, Q-deformations are used to describe these same genetic codes, and by means of a new application not considered before, it is concluded that an inverse symmetry-information relationship exists.

The work of Nikola Štambuk and Paško Konjevoda, about structural and functional modeling of artificial bioactive proteins, investigates a number of synthetic proteins designed by Michael Hecht and co-workers. The comparative investigations of the bioinformatics methods on the datasets of both de novo proteins and natural ones may lead to: (1) improvement of the existing tools for protein structure and function analysis, (2) new algorithms for the construction of de novo protein subsets, and (3) additional information on the complex natural sequence space and its relation to the individual subspaces of de novo sequences.

\subsection{Information and Symmetry in Neuroscientific and Communication Domains}

Andrew Fingelkurts and Alexander Fingelkurts analyze the information flow in the brain, considering ordered sequences of metastable states. Although Shannon's information concept has made a number of valuable contributions to neuroscience, information dynamics within the brain are not fully captured by its classical description. These additional dynamics consist of self-organization, the interplay of stability/instability, the timing of sequential processing, the coordination of multiple sequential streams, the circular causality between bottom-up and top-down operations, and information creation. In a metastable regime of brain functioning, information is continuously created, preserved for some time and then dissipated through the formation of so-called delocalized operational modules.

Arturo Tozzi and James Peters develop a symmetric approach in order to elucidate multisensory information integration. Recent advances in that field suggest that the five senses do not exist in isolation of each other. Perception, cognition and action are integrated at very early levels of central processing, in a densely coupled system equipped with multisensory interactions occurring at all temporal and spatial stages. In such a novel framework, a concept from the far-flung branch of topology, namely the Borsuk-Ulam theorem, comes into play as a general principle underlying nervous multisensory integration, resulting in a topodynamic framework that has the potential to be operationalized.

Finally, Sungchul Ji, in a very intriguing synthesis ("Waves as the Symmetry Principle Underlying Cosmic, Cell, and Human Languages"), develops the concept of Planckian information as it occurs in a number of physical and biological self-organization phenomena, implying an 'economy' of production and subsequent selection processes amidst open-ended combinatory possibilities. Further, the author concludes that living cells use a molecular language that is isomorphic with the human language, and also with a third language called the cosmic language. That which becomes common among these three kinds of languages is waves, suggested to be the symmetry principle underlying all of them as well as their medium of communication.

\section{A Special Anniversary}

With this Special Issue, we are also celebrating the 20th anniversary of the first joint meetings between Symmetrion (Symmetry Institute) and FIS (Foundations of Information Science), focused in 
the interrelationship of information and symmetry. This confluence occurred along the conferences held in Washington, DC, 1995, and Haifa, 1997, and in several subsequent venues. Different special issues in the journals Symmetry and Culture have been reflecting this cooperation. Interestingly, some of the editors and contributors in the present Special Issue of Information also appeared in those previous issues (Koichiro Matsuno, Abir Igamberdiev and Pedro Marijuán).

\section{References}

1. Anderson, P.W. More is different. Science 1972, 177, 393-396. [CrossRef] [PubMed]

2. Weyl, H. Symmetry; Princeton University Press: Princeton, NJ, USA, 1982.

3. Darvas, G. Symmetry; Birkhäuser: Basel, Switzerland, 2007.

4. Marijuán, P.C. First conference on foundations of information science: From computer and quantum physics to cell, nervous systems, and societies. BioSystems 1996, 38, 87-96. [CrossRef]

5. Igamberdiev, A.U. Information processing in biosystems: Quantum mechanical background and relation to symmetry-breaking. Symmetry Cult. Sci. 1997, 8, 193-205.

6. Stonier, T. Information and the Structure of the Universe: An Exploration into Information Physics; Springer: London, UK, 1990.

7. Conrad, M. Cross-scale information processing in evolution, development and intelligence. BioSystems 1996, 38, 87-109. [CrossRef]

8. Matsuno, K. Internalist stance and the physics of information. BioSystems 1996, 38, 111-118. [CrossRef]

9. $\mathrm{Wu}, \mathrm{K}$. The essence, classification and quality of the different grades of information. Information 2012, 3, 403-419. [CrossRef]

(C) 2017 by the authors. Licensee MDPI, Basel, Switzerland. This article is an open access article distributed under the terms and conditions of the Creative Commons Attribution (CC BY) license (http:/ / creativecommons.org/licenses/by/4.0/). 